\title{
The resurgence risk of COVID-19 in the presence of immunity waning and ADE effect: a mathematical modelling study
}

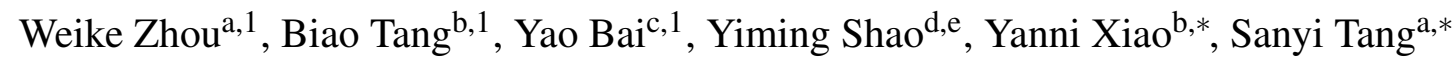 \\ ${ }^{a}$ School of Mathematics and Statistics, Shaanxi Normal University, Xi'an, 710119, P.R. China \\ ${ }^{b}$ School of Mathematics and Statistics, Xi'an Jiaotong University, Xi'an, 710049, P.R. China \\ ${ }^{c}$ Department of Infection Disease Control and Prevention, Xi'an Center for Disease Prevention and Control, \\ Xi'an, 710043, P.R. China \\ ${ }^{d}$ State Key Laboratory for Infectious Disease Prevention and Control, National Center for AIDS/STD Control and \\ Prevention, Chinese Center for Disease Control and Prevention, Beijing, 102206, P.R. China \\ ${ }^{e}$ Collaborative Innovation Center for Diagnosis and Treatment of Infectious Diseases, Hangzhou, 310058, P.R. \\ China
}

\begin{abstract}
Since the end of 2020, the mass vaccination has been actively promoted and seemed to be effective to bring the COVID-19 pandemic under control. However, the fact of immunity waning and the possible existence of antibody-dependent enhancement (ADE) make the situation uncertain. We developed a dynamic model of COVID-19 incorporating vaccination and immunity waning, which was calibrated by using the data of accumulative vaccine doses administered and the COVID-19 epidemic in 2020 in mainland China. We explored how long the current vaccination program can prevent China in a low risk of resurgence, and how ADE affects the long-term trajectory of COVID-19 epidemics. The prediction suggests that the vaccination coverage with at least one dose reach 95.87\%, and with two-doses reach 77.92\% on August 31, 2021. However, even with the mass vaccination, randomly introducing infected cases in the post-vaccination period can result in large outbreaks quickly in the presence of immunity waning, particularly for SARS-CoV-2 variants with higher transmission ability. The results showed that with the current vaccination program and a proportion of $50 \%$ population wearing masks, mainland China can be protected in a low risk of resurgence till 2023/01/18. However, ADE effect and higher transmission ability for variants would significantly shorten the protective period for more than 1 year. Furthermore, intermittent outbreaks can occur while the peak values of the subsequential outbreaks are decreasing, meaning that subsequential outbreaks boosted the immunity in the population level, which further indicating that catching-up vaccination program can help to mitigate the possible outbreaks, even avoid the outbreaks. The findings reveal that integrated effects of multiple factors, including immunity waning, ADE, relaxed interventions, and higher
\end{abstract}

\footnotetext{
${ }^{*}$ Corresponding author

Email addresses: yxiao@mail.xjtu.edu.cn (Yanni Xiao), sytang@snnu.edu.cn (Sanyi Tang)

${ }^{1}$ Equal Contributor
} 
medRxiv preprint doi: https://doi.org/10.1101/2021.08.25.21262601; this version posted August 31, 2021. The copyright holder for this preprint (which was not certified by peer review) is the author/funder, who has granted medRxiv a license to display the preprint in perpetuity.

It is made available under a CC-BY-NC-ND 4.0 International license .

transmission ability of variants, make the control of COVID-19 much more difficult. We should get ready for a long struggle with COVID-19, and should not totally rely on COVID-19 vaccine.

Keywords: COVID-19, Vaccination, Immunity waning, Antibody-dependent enhancement, Resurgence risk, Mathematical model

\section{Introduction}

Vaccination against COVID-19 has been regarded as an important measure to break the transmission chain of SARS-CoV-2 infections. Several vaccines against SARS-CoV-2 have been developed and approved by the WHO since the end of the year 2020 [1]. In mainland China, the two-doses vaccination program has been actively and widely promoted by injecting the inactivated vaccines. The vaccination of high risk populations was initiated on December 15, 2020, and over 1.82 billion COVID-19 vaccination doses had been administered by August 13, 2021 [2]. Citing the real epidemic data [3], the mass vaccination strategy seems to be able to stop the COVID-19 pandemic. However, many emerging evidences indicate that the vaccination will not help to eradicate the spread of SARS-CoV-2. On one hand, the immunity waning and the limited efficacy of the vaccines result in a large number of the vaccinated population still susceptible to SARS-CoV-2, particularly in terms of SARS-CoV-2 variants [4-6]. On the other hand, the existence of antibody-dependent enhancement (ADE) in the infection of SARS-CoV-2 has been reported recently [7].

ADE is usually referred to the phenomenon in which the pre-existing antibodies enhance the infectivity of a secondary infected virus, and facilitate the transmission of the virus. ADE phenomenon is well documented between different dengue serotypes [8-10] and Zika virus [11-13], and the infection of other corona viruses, including MERS [14] and SARS [15]. In a recent publication, Liu et al. showed that COVID-19 patients can not only produce antibodies against the RBD of the spike protein to block the SARS-CoV-2 infections, but also produce the anti-spike antibodies enhancing ACE2 binding, consequently enhancing the infectivity of SARS-CoV-2 [7]. This supports the existence of the ADE in SARS-CoV-2 infections. In [16], the author concluded two possible ways to induce the ADE effect by COVID-19 vaccines. Lots of mathematical models have been developed to discuss the impact of ADE between different dengue serotypes [17-19], or between dengue and Zika [20-22], on their transmission dynamics and viral dynamics. During the very early stage of the development of COVID-19 vaccines, several researchers posted their concerns on that ADE can be a potential safety issue [23, 24]. However, it remains unclear and challenging how the ADE effect in SARS-CoV-2 infection will affect the trajectory of the COVID-19 pandemic when we use COVID-19 vaccines in the population.

What's more, Choe et al. conducted a clinical study to measure the changes of neutralizing antibodies in both symptomatic and asymptomatic SARS-CoV-2 infection, and found that the geometric mean titer of neutralizing antibodies declined from 219.4 at 2 months to 143.7 at 5 months after infection [5]. Similarly, in [6], based on a longitudinal study of 517 COVID-19 
patients, the authors observed different levels of immunity waning after symptoms onset. The immunity waning makes the prospect of achieving herd immunity increasingly remote, that is, the prominence of herd immunity being touted as a solution to the pandemic might be about to change [25]. Therefore, it's urgent to evaluate the impact of the immunity waning on the trends of COVID-19 epidemics, and it's essential to re-design the optimal control interventions in combating with COVID-19 pandemic in a long-term. This also remains challenging.

Hence, the immunity waning and ADE make the long-term trajectory of COVID-19 epidemics be full with uncertainty. This study aims to develop a mathematical model describing the transmission process of COVID-19 and the two-doses vaccination program incorporating with the immunity waning and ADE effect, to investigate the effect of immunity waning and ADE. We used the COVID-19 epidemic data from January 23 to April 8, 2020 and cumulative vaccine doses administered in mainland China to inform model parameters and conducted sensitivity analysis to evaluate how long the program can protect China in a low risk of resurgence, and how ADE will affect the transmission dynamics of COVID-19 epidemics. Findings from this study will provide important information for policymakers on the critical time of implementing strict control measures and when the catch-up vaccination program should be launched.

\section{Methods}

We proposed a modelling framework which incorporates the infection and transmission process of COVID-19 with the two-doses vaccination program and the immunity waning. The model can be reduced to a transmission model only and a vaccination model only to estimate the parameters related with the transmission and the vaccination by using epidemic data and vaccination data. The full model was used to simulate different scenarios during the postpandemic period to evaluate the resurgence risk and explore the low risk protective period.

\subsection{Model overview}

We developed a dynamic model of COVID-19 infection and transmission incorporating with the vaccination program and immunity waning in mainland China (Fig. 1 and model (1) in SI). The modelling framework SEIARS is used to describe the transmission dynamics, where the route that recovered individuals $R$ can go back to susceptible population $S$ is used for modelling the immunity waning. The population is further divided into three categories according to their vaccination states: not vaccinated, vaccinated by one-dose, vaccinated by two-doses. Only part of the vaccinated population is effectively protected, hence the vaccinated but not effectively protected population also evolve a transmission dynamic with the same structure of SEIARS. Similar to the recovered population, individuals either effectively protected by the first dose or by two-doses vaccine will also lose their immunity after a period of time. It should be mentioned that the recovered population have the same level of immunity as those effectively protected by two-doses. Given the possibility of the existence of ADE, we assumed that the susceptibility of the individuals lost their immunity is higher than those without any immunity 
before. In particular, $\kappa$ is denoted as the modification factor of the susceptibility. The detailed assumptions and the corresponding model equations are shown in SI.

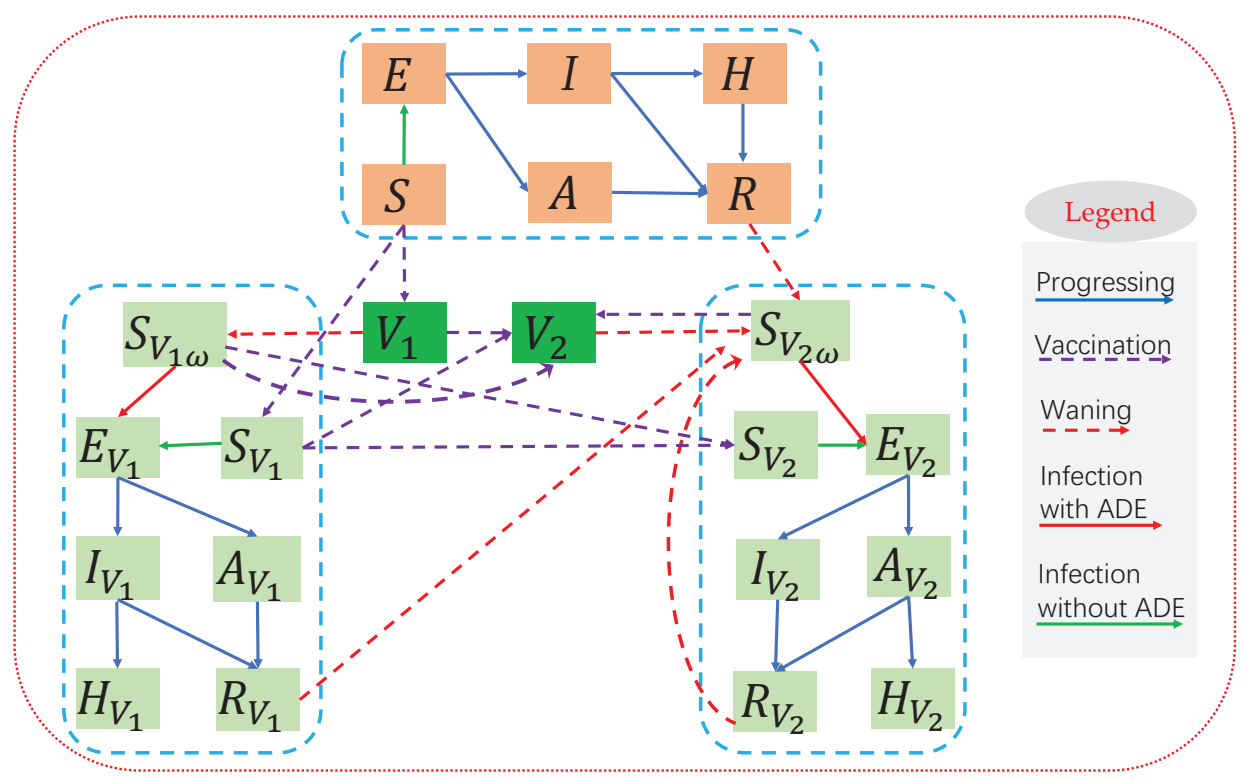

Figure 1: Schematic diagram to illustrate the transmission of COVID-19 incorporating with the vaccination program and immunity waning.

\subsection{Data}

We obtained the data of the COVID-19 epidemic, and the data of the mass vaccination program in mainland China from the National Health Commission of the People's Republic of China [2] and Our World in Data [26]. The epidemic data includes the number of daily confirmed cases and deaths from January 23, 2020 to April 8, 2020 in mainland China, as shown in Fig. 2 (a) and 2 (b). The data of the vaccination program, collected from December 15, 2020 to June 29, 2021, contains the cumulative vaccine doses administered and the daily vaccine doses administered in mainland China, see Fig. 2(c) and 2(d). It should be mentioned that the vaccination data is available from December 15, 2020 with a report of accumulative $1,500,000$ doses used in the population. However, during the period from December 15, 2020 to March 23, 2021, the data was not released daily, hence is intermittent. Since March 23, 2021, the vaccination data was reported per day.

\subsection{Model calibration}

To calibrate the model, we firstly use the data of the COVID-19 epidemic from January 23, 2020 to April 8, 2020 in mainland China to estimate the parameters related to transmission dynamics. As there is no vaccination during this period, the full model can be reduced to a 

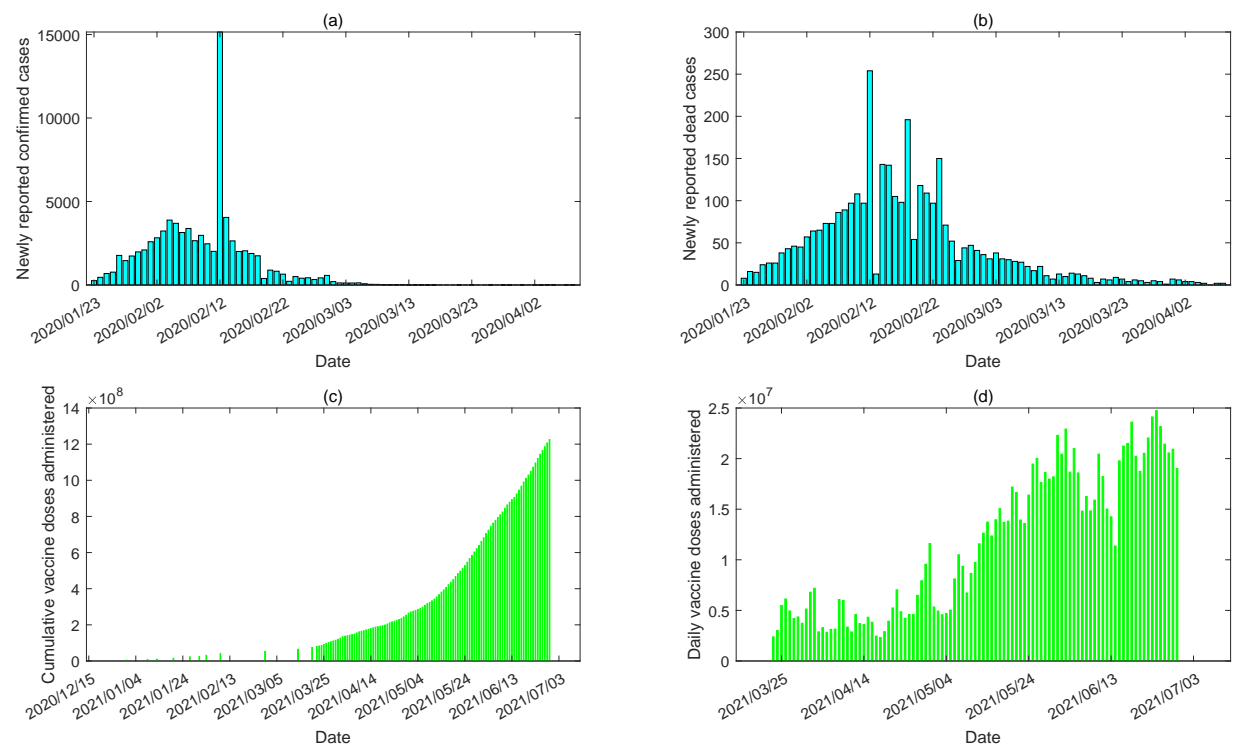

Figure 2: The epidemic data of COVID-19 in mainland China from January 23, 2020 to April 8, 2020 ((a)-(b)), and the vaccine doses administered in mainland China from December 15, 2020 to June 29, 2021 ((c)-(d)). (a) Daily reported confirmed cases; (b) Daily reported dead cases; (c) Cumulative vaccine doses administered; (d) Daily vaccine doses administered.

model without vaccination (i.e. model (3) in SI). Note that, because the epidemic lasted less than four months and only a very small proportion of the whole population was infected, we didn't consider the immunity waning in the reduced model. Considering the continuously enhanced control interventions implemented by the government, we introduced a time-dependent transmission rate and diagnose rate. Based on these assumptions, we fit model (3) to the epidemic data of mainland China in 2020. To this end, we first fixed several parameters from the literature and initial values from the database, as listed in Table 1 . Given the randomness of reported cases, we used a bootstrap method to generate 1000 time series of daily confirmed cases and deaths from a Poisson distribution with mean given by the reported data, hence we obtained 1000 data set. We then use the least square method to fit the model to each data set.

We further used the vaccination data in mainland China from December 15, 2020 to June 29, 2021 to estimate the parameters related to the mass vaccination program in China. To this end, we deduced a vaccination dynamical model (model (6) in SI) from the full modelling framework. Considering the initial accessability of vaccines and the capacity of daily vaccination population, we set the rate, at which the population receive the first dose, as a logistic function of time. In contrast, the rate at which the individuals get the second dose is fixed as a constant. Note that the second doses is requested to be vaccinated in 3-8 weeks in China [27], hence we set $v_{2}=\frac{1}{35}$. Based on the vaccination data, the initial time is set as December 15, 2020, and the initial conditions are $S(0)=1,400,000,000, S_{1}(0)=1,500,000, S_{2}(0)=0$. Then, by using the similar methods as in fitting the epidemic data, we fitted the vaccination dynamic model to 
1000 vaccination data set.

Table 1: Definitions and values of variables and parameters

\begin{tabular}{|c|c|c|c|c|}
\hline \multicolumn{2}{|c|}{ Variables } & Description & Initial value & Resource \\
\hline \multicolumn{2}{|c|}{$S$} & Susceptible population & $1.4 \times 10^{9}$ & {$[28]$} \\
\hline \multicolumn{2}{|l|}{$E$} & Exposed population & 5926 & LS \\
\hline \multicolumn{2}{|l|}{$I$} & Symptomatic infected population & 3591 & LS \\
\hline \multicolumn{2}{|l|}{$A$} & Asymptomatic infected population & 3459 & $\mathrm{LS}$ \\
\hline \multicolumn{2}{|l|}{$H$} & Hospitalized population & 771 & data \\
\hline \multicolumn{2}{|l|}{$R$} & Recovered population & 34 & data \\
\hline \multicolumn{2}{|c|}{ Parameters } & Description & Value & Resource \\
\hline \multicolumn{5}{|c|}{ Parameters related to the disease transmission } \\
\hline \multirow{3}{*}{$\beta(t)$} & $\beta_{0}$ & Transmission rate in the pre-pandemic era & 2.8749 & LS \\
\hline & $\beta_{1}$ & Minimum transmission rate with control strategies & 0.0521 & LS \\
\hline & $r_{b}$ & $\begin{array}{l}\text { Exponential decreasing rate of the transmission } \\
\text { rate }\end{array}$ & 0.1232 & $\mathrm{LS}$ \\
\hline \multicolumn{2}{|l|}{$\theta$} & Relative transmissibility of $A$ to $I$ & 0.55 & [29] \\
\hline \multicolumn{2}{|l|}{$\sigma$} & $\begin{array}{l}\text { Progression rate of exposed individuals to infec- } \\
\text { tives }\end{array}$ & $1 / 5.2$ & [30, 31] \\
\hline \multirow[t]{2}{*}{$\rho$} & & Probability of symptomatic & 0.4972 & LS \\
\hline & $\delta_{I_{0}}$ & Initial diagnose rate of infected individuals & 0.1 & [29] \\
\hline \multirow[t]{2}{*}{$\delta_{I}(t)$} & $\delta_{I_{1}}$ & Maximum diagnose rate of infected individuals & 0.5848 & LS \\
\hline & $r_{d}$ & $\begin{array}{l}\text { Exponential decreasing rate of diagnose rate from } \\
\text { symptom onset to detection }\end{array}$ & 0.1247 & LS \\
\hline \multicolumn{2}{|l|}{$\gamma_{I}$} & Recovery rate of symptomatic infectives & $1 / 2.9$ & [29] \\
\hline \multicolumn{2}{|l|}{$\gamma_{A}$} & Recovery rate of asymptomatic infectives & $1 / 2.9$ & [29] \\
\hline \multicolumn{2}{|l|}{$\gamma_{H}$} & Recovery rate of hospitalized infectives & 0.2149 & LS \\
\hline \multicolumn{2}{|l|}{$\alpha$} & Disease induced death rate & 0.0087 & LS \\
\hline \multicolumn{5}{|c|}{ Parameters related to the vaccination program and immunity waning } \\
\hline \multirow{3}{*}{$v(t)$} & $v_{0}$ & Initial vaccination rate of the first dose & $5.0437 \times 10^{-5}$ & LS \\
\hline & $v_{1}$ & Minimum vaccination rate of the first dose & 0.0585 & LS \\
\hline & $r_{v}$ & $\begin{array}{l}\text { Net increasing rate of the vaccination rate of the } \\
\text { first dose }\end{array}$ & 0.0338 & $\mathrm{LS}$ \\
\hline \multicolumn{2}{|l|}{$v_{2}$} & Vaccination rate of the second dose & $1 / 35$ & [27] \\
\hline \multicolumn{2}{|l|}{$p_{1}$} & Effective protection rate to $S$ by one-dose vaccine & 0.3 & {$[32-34$} \\
\hline \multicolumn{2}{|l|}{$p_{2}$} & $\begin{array}{l}\text { Effective protection rate to } S_{V_{1}} \text { by two-doses vac- } \\
\text { cine }\end{array}$ & 0.9 & {$[32-34$} \\
\hline \multicolumn{2}{|l|}{$p_{3}$} & $\begin{array}{l}\text { Effective protection rate to } S_{V_{1 \omega}} \text { by two-doses vac- } \\
\text { cine }\end{array}$ & 0.9 & Assumed \\
\hline \multicolumn{2}{|l|}{$\omega_{i}$} & Immunity waning rate $\left(i=R, R_{V_{1}}, R_{V_{2}}, V_{1}, V_{2}\right)$ & $1 / 365$ & Assumed \\
\hline \multicolumn{2}{|l|}{$\kappa$} & Modification factor of susceptibility & {$[1,3]$} & $\begin{array}{l}{[17-19,35} \\
36]\end{array}$ \\
\hline
\end{tabular}




\subsection{Parameter setting for simulations}

Because of the highly improved testing capacity, we set the diagnose rate as the estimated maximum rate (i.e. $\delta_{I}=\delta_{I_{1}}$ ) when carrying the simulations. The baseline protective rate of the first dose and the second dose vaccine are $p_{1}=0.3, p_{2}=0.9$, respectively [32-34]. Suppose the immunity produced by infection or vaccination lasts 1 year on average, then the immunity waning rate $\omega_{R}=\omega_{V_{1}}=\omega_{V_{1}}=\omega_{R_{V_{1}}}=\omega_{R_{V_{2}}}=\omega=1 / 365$ per day. Using face mask is recognized as an useful self-protective way to prevent the COVID-19 infection. During the COVID-19 epidemic in 2020 in China, the proportion of people wearing face masks increase from $0 \%$ to almost $100 \%$, thus face mask use would be a normalized prevention and control intervention. Assume that the baseline proportion of face mask use is around $50 \%$ in the postpandemic era, and the effectiveness of face mask in preventing COVID-19 infection or infecting others is $80 \%$ based on a recent meta-analysis on the effectiveness of face mask use [37]. Thus the baseline transmission rate with a normalized control intervention of wearing masks would be $(1-50 \% \times 80 \%) \beta_{0}=60 \% \beta_{0}$. Given the enhanced intervention, the transmission rate can decrease further, and considering the higher transmission ability of SARS-CoV-2 variants, the transmission rate can also be higher than the initial value $\beta_{0}$. Consequently, when performing sensitivity analysis, we choose the transmission rate from $0.4 \beta_{0}$ to $1.5 \beta_{0}$. In the absent of real data, we pick up a range of $[1,3]$ for the modification factor of $\operatorname{ADE}(\kappa)$ from the studies on the ADE in dengue infections [17- 19, 35, 36].

\section{Main results}

\subsection{Estimation results}

The fitting results of the transmission dynamic model (model (3) with (4) and (5) in SI) to the epidemic data of mainland China in 2020 are shown in Fig. 3, with the best fitting curves marked as black. Based on the fitting results, we obtained the estimation of the unknown parameters (listed in Table 1), and also the estimated effective reproduction number (Fig. 3(c)). The fitting results of the vaccination dynamic model (model (6) with (7) in SI) to the vaccination data are shown in Fig. 4. And we obtained the estimation of the vaccination rates, as listed in Table 1. The estimation showed that the population vaccinated with at least one dose vaccine reached $56.4 \%(95 \% \mathrm{CI}[55.38 \%, 57.08 \%])$ while the population vaccinated with both two doses reached $32.02 \%$ (95\% CI [31.93\%, 32.06\%]) on June 29, 2021 (the last data point). A further prediction shows that the population vaccinated with at least one dose can reach $95.87 \%$ (95\% CI [91.12\%,98.16\%]) and people fully vaccinated reaches $77.92 \%(95 \% \mathrm{CI}[73.33 \%, 79.33 \%])$ on August 31, 2021. As we can see, the vaccination coverage in China is very high till August 31, 2021, hence we assume to stop the routine vaccination program on August 31, 2021. Of course, we should complete the second dose injection for individuals who has been given the first dose already. Note that, unless otherwise stated, the considered time period is always to the end of year 2022 when performing the simulations. 

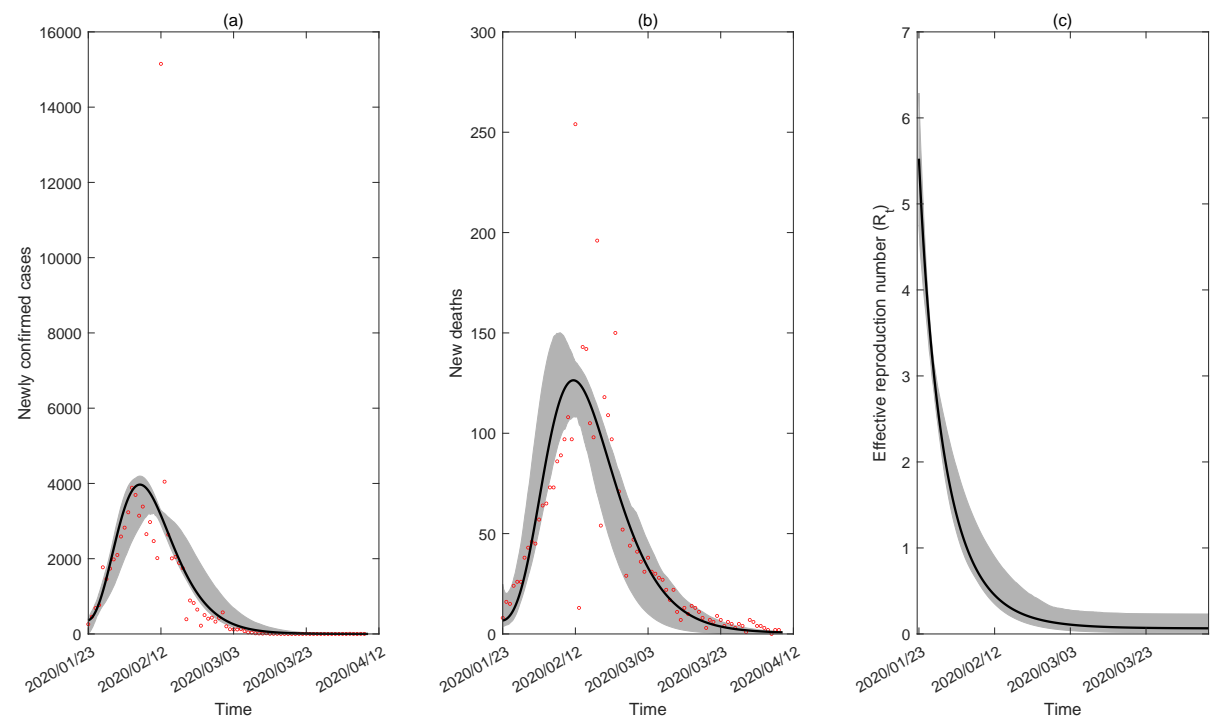

Figure 3: Model fitting results for the transmission dynamic model in mainland China in 2020. (a) The daily reported confirmed cases. (b) The daily reported dead cases. (c) The estimated effective reproduction number. The black curves are the estimated curves with the shadow areas as the corresponding $95 \%$ confidence intervals. The red circles in (a) and (b) are the observed data of the daily reported confirmed cases and the daily reported dead case from January 23, 2020 to April 8, 2020 in mainland China. 

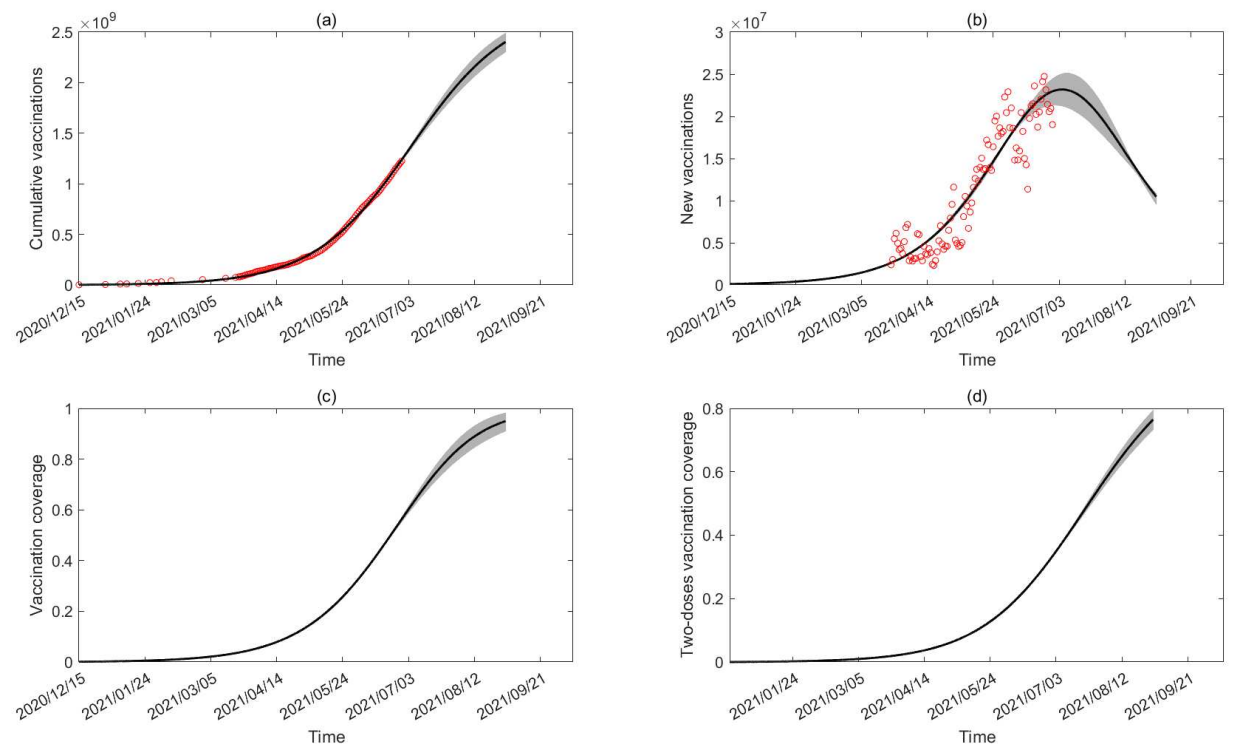

Figure 4: Model fitting results for the vaccination dynamic model. (a) The accumulative number of vaccine doses administered. (2) The daily number of vaccine doses administered. (c) Proportion of population vaccinated with at least one dose. (d) Proportion of population vaccinated with both two doses. The black curves are the estimated curves with the shadow areas as the corresponding $95 \%$ confidence intervals. The red circles in (a) and (b) are the reported data of the accumulative vaccine doses and daily vaccine doses administered from December 15,2020 to June 29, 2021 in mainland China. 


\subsection{Resurgence risk evaluation}

Based on the above estimation results, through numerical simulations, we focused on discussing the impact of immunity waning and ADE effect on the transmission dynamics of COVID-19, and evaluating the resurgence risk of COVID-19 in China. As we know, the COVID-19 epidemic in China is almost under control. The biggest challenge is that the imported cases have caused several local outbreaks in China. Therefore, we analyzed if there can be an large outbreak only with the mass vaccination or the vaccination plus a normalized control intervention by wearing masks, by randomly introducing several infected cases into the community.

Assume that 10 infected cases are introduced on September 1, 2021, Fig. 5 shows the number of newly confirmed cases and the effective reproduction number $R_{t}$ during the transmission process, with different transmission rate and various ADE degree. It follows from Fig. 5(a) and 5(c) that, even without ADE $(\kappa=1)$, introducing infected cases would cause the large outbreaks (black curves) as the immunity waning. Worse still, ADE would facilitate the outbreak by bringing forward the peak time and increasing the peak value. Comparing Fig. 5(a) and Fig. 5. $(\mathrm{c})$, we find that the normalized intervention $\left(\beta=0.6 \beta_{0}\right)$ cann help to delay the outbreak and reduce the peak value. We also observed several subsequential epidemic waves in Fig. 5(a) and 5(c), while the peak values of the subsequential waves are decreasing. Furthermore, the $\mathrm{ADE}$ effect and the higher transmission ability can also increase the frequency of the outbreaks. Correspondingly, the effective reproduction number fluctuates around the threshold of unit, as shown in Fig. 5(b) and 5(d).
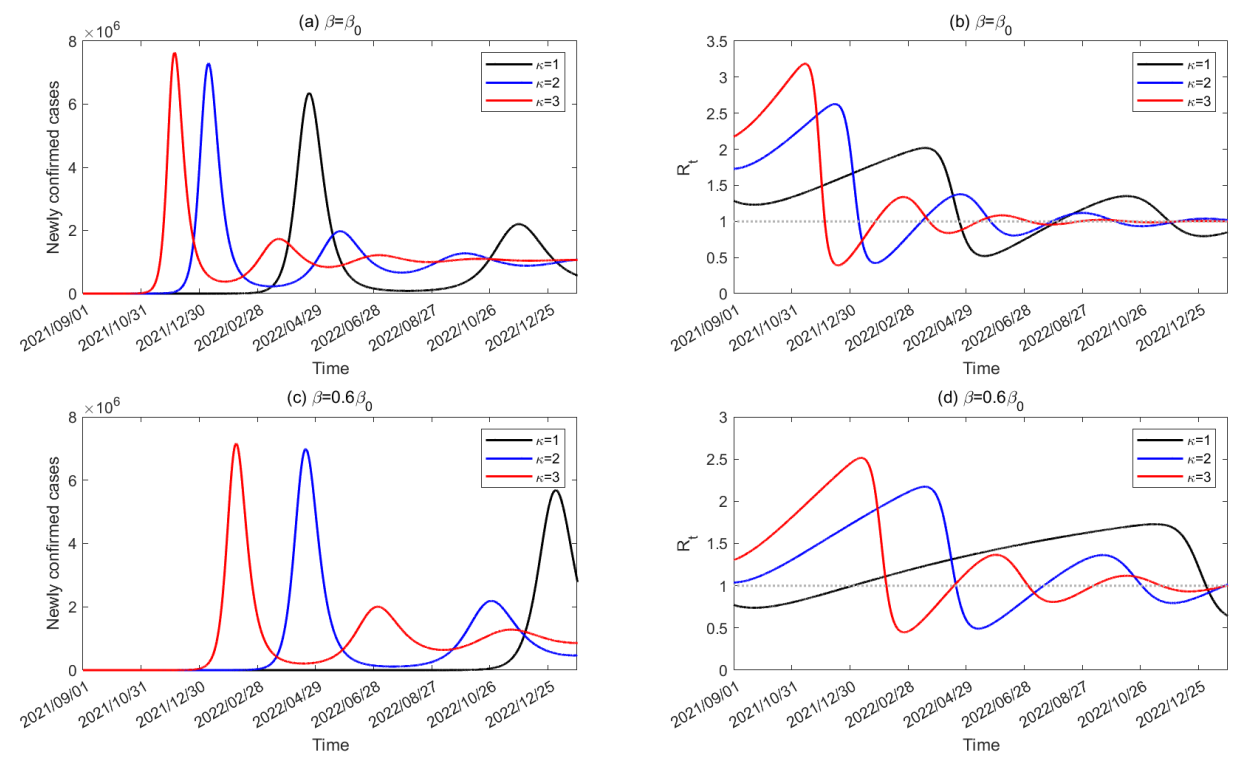

Figure 5: Effect of the ADE and the control interventions on the number of newly confirmed cases and effective reproduction number during the transmission process when introducing 10 infected cases on September 1, 2021. 
In Fig. 5, we always set the introducing time as September 1, 2021, we then explored the impact of the introducing time on the transmission dynamics of COVID-19. To this end, by assuming the introducing time to be September 1, 2021, November 1, 2021, January 1, 2022, respectively, we further plotted the number of newly confirmed cases and effective reproduction number with a normalized control intervention $\left(\beta=0.6 \beta_{0}\right)$ in Fig. 6. Note that, although we chose different introducing date, we run the same transmission period of 500 days. From Fig. 6(a) and 6(c), we can see that the later of the introducing date, the time length that the outbreak takes to peak is shorter. Basically, the reason is that the reproduction number at the initial stage for the introducing time of Jan 1, 2022 is higher than those for the introducing time of Nov 1 and Sep 1, 2021, as shown in Fig. 6(b) and 6(d). On the other hand, we observed an interesting phenomenon that when $\kappa=1$, the earlier introducing date caused larger outbreak while when $\kappa=2$, the later introducing date caused larger outbreak. Therefore, the peak value of the outbreak is non-monotonous with respect to the introducing time, which is dependent on the ADE effect. Without ADE (i.e. $\kappa=1$ ), a faster growth rate (i.e. the higher effective reproduction number initially) lead to smaller outbreak, as shown in Fig. 6(a) and 6(b). However, when $\kappa=2$, a faster infection process means that more population gained the immunity and then lost their immunity, consequently, more susceptible population with ADE effect can be reinfected with a higher peak value (Fig. 6(c) and 6(d)). Therefore, the introducing time can also have a great impact of the transmission dynamics of COVID-19 in the presence of immunity waning.
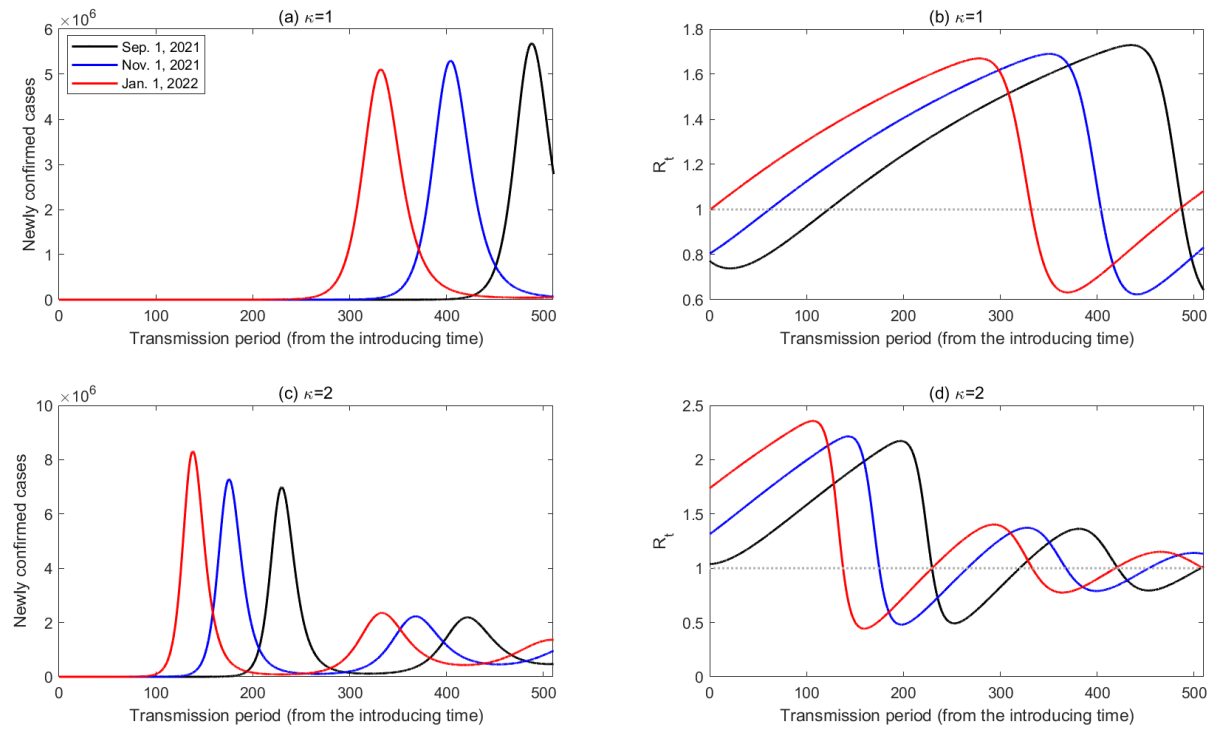

Figure 6: Effect of the ADE and different introducing date on the number of newly confirmed cases and the effective reproduction number during the transmission period.

From the above analysis, we can see that the introducing time can have a great impact on the initial reproduction number because of the immunity waning. Note that, before the introducing 
date, there is no infected case, hence no transmission in the population. Therefore, the changing of the initial reproduction number totally depends on the immunity waning dynamics without the transmission dynamics. For this reason, we define a new reproduction number as $R_{s}=R(s)$, which denotes the reproduction number at the introducing time $s$. In Fig. 7(a) and 7(b), we plotted changing curves of $R_{S}$ by choosing different transmission rate $\beta$, ADE factor $\kappa$ and waning rate $\omega$. We can easily see from Fig. 7(a) and 7(b) that $R_{S}$ is increasing over time because of the immunity waning. On the other hand, with a higher transmission ability or ADE degree or immunity waning rate, the reproduction number at the introducing time is always greater, as shown in Fig. 7(a) and (b). By plotting the partial rank correlation coefficients (PRCCs) [38], we also conducted a sensitivity analysis of $R_{S}$ with resect to the transmission related parameters $\left(\beta, \rho, \delta_{I}, \theta, \gamma_{I}, \gamma_{A}\right)$ and vaccination related parameters $\left(p_{1}, p_{2}, p_{3}, \omega_{V_{1}}, \omega_{V_{2}}\right)$ and the ADE factor $\kappa$ over time, as shown in Fig. 7(c). As a results, we found that the transmission rate always have the most significant effect and is positive related to $R_{s}$. $\kappa$ does not dominant before June 13, 2021, which means that ADE almost has no effect in the early stage of the vaccination program, mainly due to the high effectiveness of the vaccine and majority of the population had not been vaccinated. However, $\kappa$ positively affects the reproduction number significantly after June 13 , 2021, attributing to the fact that people vaccinated may lose the immunity and has the ADE after a period of time. In particular, the PRCCs with respect to the vaccination parameters and the ADE factor (Fig.7p(d) showed that the immunity waning rate $\omega_{V_{1}}$ of those received the first dose is also positive related to $R_{s}$, which is high in the initial stage of the vaccination program. The PRCC of the immunity waning rate $\omega_{V_{2}}$ is high in the middle stage of the vaccination program, and the PRCC of the efficacy of two-doses $p_{2}$ becomes high in the late stage, illustrating the evolution of the vaccination and immunity waning dynamics.

In conclusion, we have defined two time-varying reproduction numbers $R_{t}$ and $R_{s}$. Here, we tried to combine the two time-varying reproduction number together, and denoted it as $R(t, s)$, where $s$ is the introducing time since the initial time and $t$ is the transmission period since the introducing time. Therefore, we have that $R_{s}=R(0, s)$ denotes the reproduction number at the introducing time $s$ while $R_{t}=R(t, 0)$ is the traditional effective reproduction number at time $t$, when the introducing time is taken as the initial time of the epidemic. With this definition, we can easily check the effective reproduction numbers of an epidemic starting at different time. In particular, Fig. 8 show the contour plot of $R(t, s)$ with respect to different introducing time $s$ (taking September 1, 2021 as the initial introducing time) and the transmission period $t$ (taking the introducing time as initial time of the transmission process), with the baseline transmission rate $\beta=0.6 \beta_{0}$ and the ADE factor $\kappa=1$ and $\kappa=2$, respectively. From Fig. 8 , we can easily see that $\kappa$ magnify $R(0, s)$ and makes $R(t, s)$ tend to be stabilized more quickly. Furthermore, $R(0, s)$ increases as $s$ increases, and $R(t, s)$ increase first and then fluctuates around the unit with respect to $t$ given arbitrary introducing time $s$, which is also observed in Fig. 5 and 6 .

\subsection{Protective period evaluation and analysis}

Usually, the effective reproduction number is taken as the only risk index to show if the epidemic is under control or not. That is because the new infection will decrease when the 

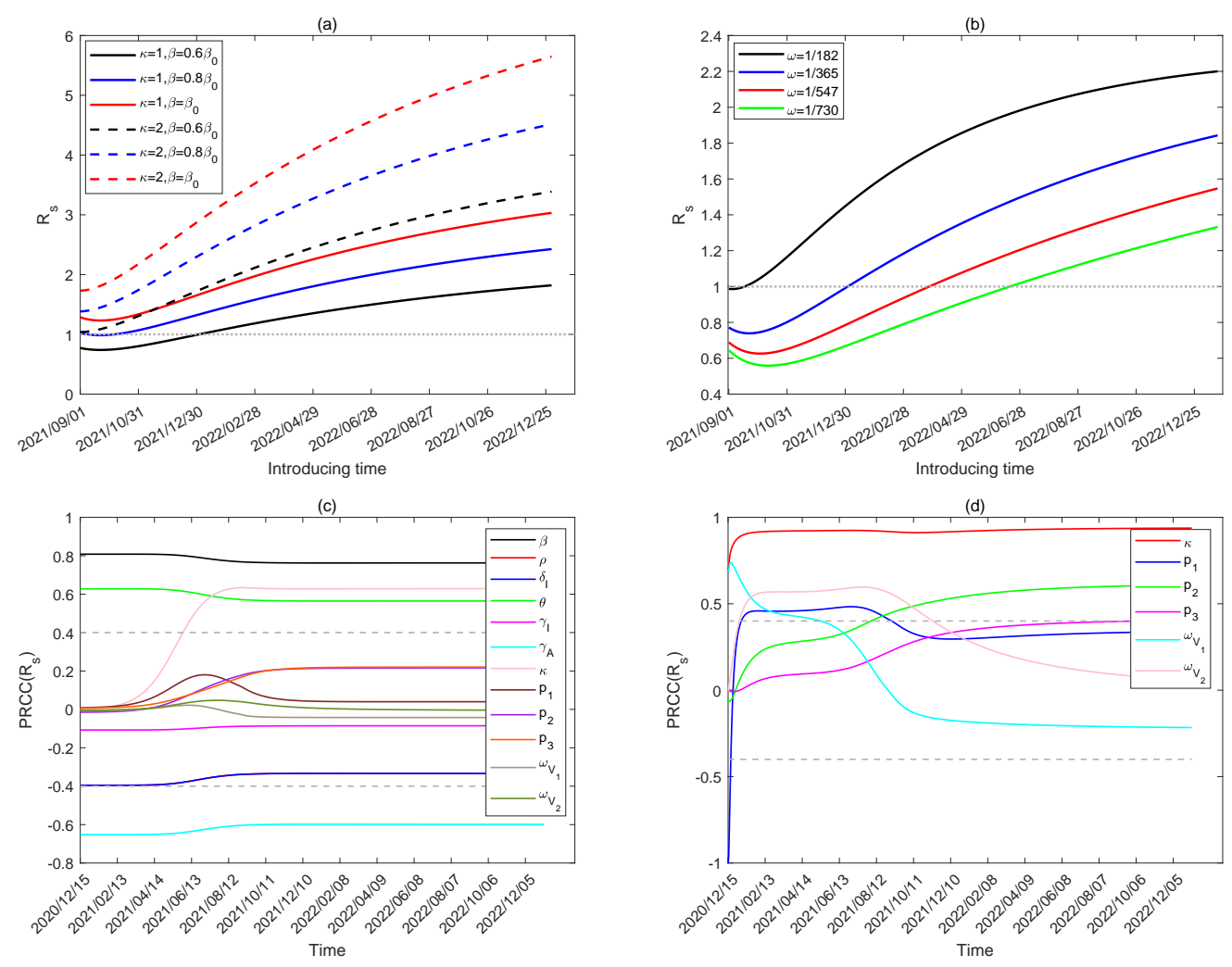

Figure 7: (a) Effect of $\beta$ and $\kappa$ on the initial reproduction number $R_{s}$. (b) Effect of $\omega$ on the initial reproduction number $R_{s}$. (c) Partial rank correlation coefficients (PRCCs) of the initial reproduction number $R_{s}$ at different introducing time $s$ with respect to parameters related to the disease transmission dynamics $\left(\beta, \rho, \delta_{I}, \theta, \gamma_{I}, \gamma_{A}\right)$ and parameters related to the vaccination program $\left(\kappa, p_{1}, p_{2}, p_{3}, \omega_{V_{1}}, \omega_{V_{2}}\right)$. (d) Partial rank correlation coefficients (PRCCs) of the initial reproduction number $R_{s}$ at different introducing time $s$ with respect to the ADE factor $(\kappa)$, vaccination efficacy $\left(p_{1}, p_{2}, p_{3}\right)$, and vaccination protective period $\left(\omega_{V_{1}}, \omega_{V_{2}}\right)$. Other parameters are fixed as in Table1.
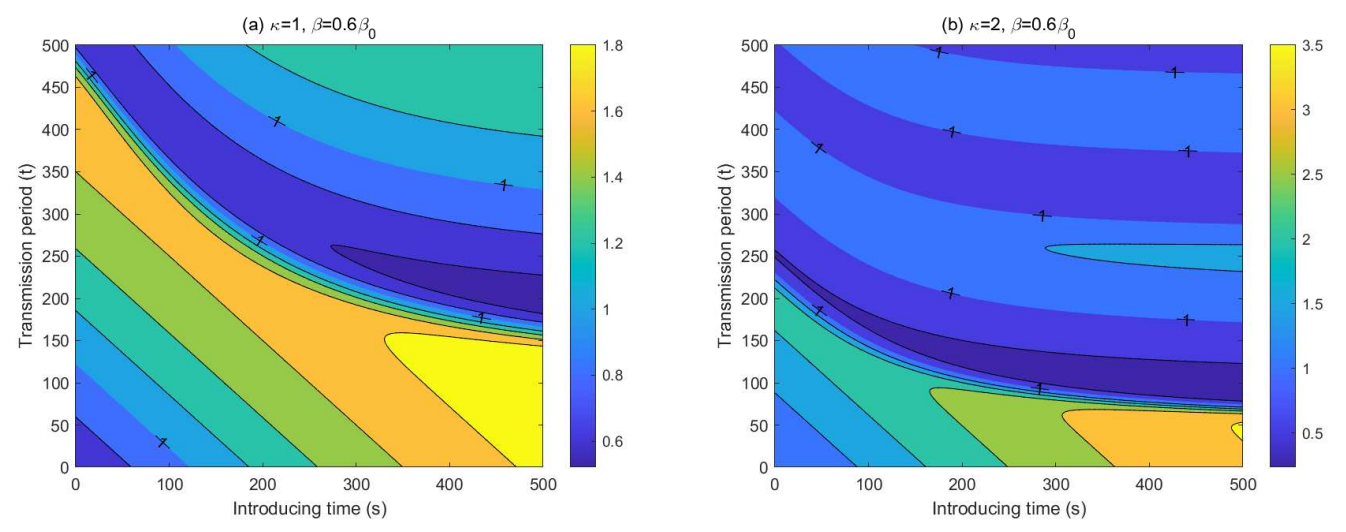

Figure 8: Values of $R(t, s)$ with different introducing time $s$ (taking September 1, 2021 as the initial introducing time) and transmission period $t$ (taking the introducing time as initial time of the transmission process). 
effective reproduction number is less than the unit. However, as we illustrated in [39], that the effective reproduction number is less than 1 does not mean that the epidemic is totally under control or we can finally achieve the goal of no newly reported case. It will take a long period to achieve the goal of no newly reported cases. During this period, because of the existence of the source of infection, the COVID-19 epidemic can easily boost once the normalized control interventions are released. Similarly, we cannot say that it will be out of control when the effective reproduction number is greater than the unit when several infected cases are introduced into the community. Therefore, we provide a new definition to represent when an emerging outbreak of COVID-19 can be under control or of a low level of risk.

For any given number of infected cases $\left(I_{0}\right)$ being introduced into the community, it's reasonable to say that if the newly reported cases is always lower than $I_{0}$ (or higher than $I_{0}$ but lower than $5 I_{0}$ ) in the next 30 days, it's of a low (or medium) level of risk in terms of the introducing time. Otherwise, we say that it's of a high level of risk. Based on the definition, we can further define a critical time $T_{1}$ before which the new epidemic is in a low level of risk if several cases are introduced into the community. That is, $T_{1}$ is the first time at which several cases are introduced into the community, and the newly reported cases will reach $I_{0}$ at the 30th day. Similarly, we can define the critical time $T_{2}$, at which several cases are introduced into the community, and the newly reported cases will reach $5 I_{0}$ at the 30 th day. Consequently, for the introducing time before $T_{1}$ (or between $T_{1}$ and $T_{2}$, or after $T_{2}$ ), the epidemic is in a low (or medium or high) level of risk. Note that, we observed an interesting phenomenon that for any given number of infected cases $I_{0}$ introduced at time $s$, the time length it takes for the number of newly confirmed cases increasing to $k I_{0}(k \geq 1)$ is independent of the value of $I_{0}$, see a theoretical illustration in SI. This means that the critical time $T_{1}$ and $T_{2}$ is also independent of the number of cases introduced into the community.

In Fig. 9, we plotted the time length that it takes for the number of newly confirmed cases to reach $I_{0}$ or $5 I_{0}$ since the introducing time, respectively. Thus, the intersection points of the curves and the horizontal dash line are actually the critical time $T_{1}$ or $T_{2}$. It follows from Fig. 9(a) and 9.b) that the later introducing of infected cases, the shorter time length it takes for the number of newly confirmed cases increasing to $I_{0}$ or $5 I_{0}$. Comparing the dash or solid curves with different colors in Fig. 9(a) or 9(b), we find that the increases of the transmission rate and the ADE degree will bring forward the critical time $T_{1}$ and $T_{2}$, consequently shorten the time period during which the epidemics is of low risk, and bring forward the time after which the epidemic is of high risk by introducing several infected cases. In particular, in the baseline situation, i.e. $\kappa=1, \beta=0.6 \beta_{0}$, introducing infected cases before the end of the year 2022 would not lead to a large outbreak quickly (in a low level of risk). That is, in this situation, the emerging outbreak by introducing infected cases is always in a low risk till December 31, 2022. When the transmission rate increases to $0.8 \beta_{0}$ or $\beta_{0}$, corresponding to the release of normalized control interventions, the emerging outbreak is in the low level of risk, by the cases introduced before April 28, 2022 or January 22, 2022, respectively.

In addition, further considering the higher transmission ability of the SARS-CoV-2 variants, we plotted the time length it takes for the confirmed cases to reach $I_{0}$ or $5 I_{0}$ since the introducing 
time in Fig. 9 (c) and (d), by choosing the transmission rate as $1.2 \beta_{0}$ and $1.5 \beta_{0}$. It follows from Fig. 9(c) that when $\kappa=2, \beta=1.2 \beta_{0}$ or $\kappa=2, \beta=1.5 \beta_{0}$, the curves are always below the horizontal dash line since September 1, 2021. This means that if the ADE is possible, the emerging outbreak of SARS-CoV-2 variants can not be in a low level of risk by introducing new infected cases since September 1, 2021, and it's in a high level of risk since the initial time we considered by introducing infected cases when $\kappa=2, \beta=1.5 \beta_{0}$ (Fig. 9(d)). On the other hand, if we fix $\beta=0.6 \beta_{0}$ but assume that that the ADE is possible by letting $\kappa=2$, the critical time $T_{1}$ and $T_{2}$ becomes January 4, 2022, and March 25, 2022, respectively, as shown in Fig. 9(a),(b) and Table 2. To summary, Table 2 lists the critical time $T_{1}$ and $T_{2}$ under the different situations with different transmission rate and ADE degree.
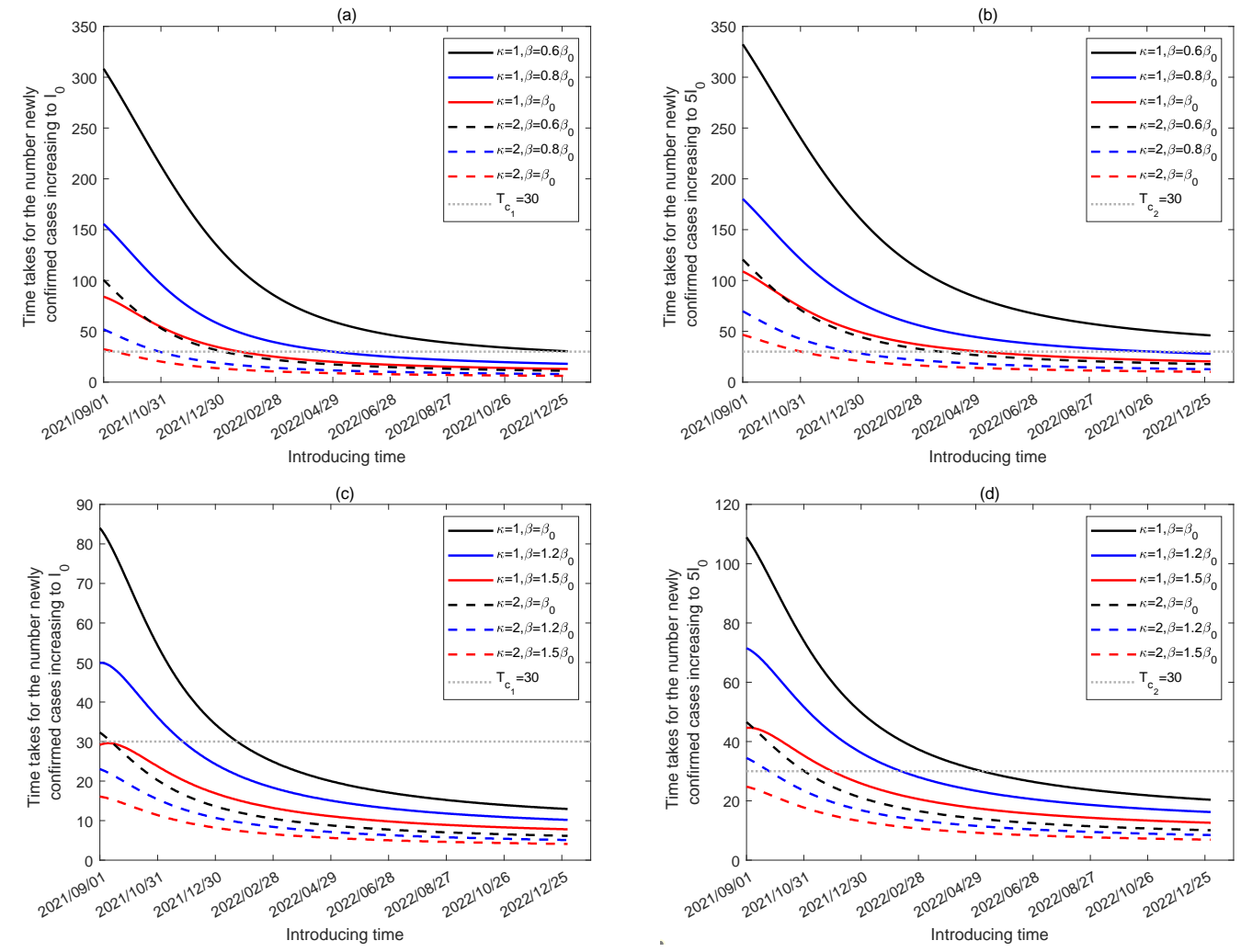

Figure 9: Length of time it takes for the newly confirmed cases increasing to $I_{0}$ and $5 I_{0}$, respectively, when introducing infected cases at different times for different $\beta$ and $\kappa$.

In addition, we showed the contour plots of $T_{1}$ and $T_{2}$, respectively, by regarding September 1, 2021 as the initial time 0 in Fig. 10, with respect to the transmission rate $\beta$ and the ADE factor $\kappa$ (Fig. 10 (a) and (b)), and with respect to the transmission rate $\beta$ and the waning rate $\omega$ (Fig. 10(c) and (d)). From Fig. 10 (a) and (b) we can see that $T_{1}$ and $T_{2}$ decreases with the increase of $\beta$ and $\kappa$, meaning that the low risk period is shorten and the high risk time is brought forward. $T_{2}$ is certainly greater than $T_{1}$ while for variants with high transmission ability and 
Table 2: The impact of the transmission rate and ADE factor on the time period of the low, medium and high risk levels by introducing infected cases.

\begin{tabular}{llll}
\hline Parameters & Low risk period & Medium risk period & High risk period \\
\hline$\kappa=1, \beta=0.6 \beta_{0}$ & before 2023/01/18 & - & - \\
$\kappa=1, \beta=0.8 \beta_{0}$ & before 2022/04/28 & $2022 / 04 / 28-2022 / 11 / 01$ & after 2022/11/01 \\
$\kappa=1, \beta=\beta_{0}$ & before 2022/01/22 & $2022 / 01 / 22-2022 / 05 / 05$ & after 2022/05/05 \\
$\kappa=1, \beta=1.2 \beta_{0}$ & before 2021/11/26 & $2021 / 11 / 26-2022 / 02 / 10$ & after 2022/02/10 \\
$\kappa=1, \beta=1.5 \beta_{0}$ & - & before 2021/11/29 & after 2021/11/29 \\
\hline$\kappa=2, \beta=0.6 \beta_{0}$ & before 2022/01/04 & $2022 / 01 / 04-2022 / 03 / 25$ & after 2022/03/25 \\
$\kappa=2, \beta=0.8 \beta_{0}$ & before 2021/10/30 & $2021 / 10 / 31-2021 / 12 / 21$ & after 2021/12/21 \\
$\kappa=2, \beta=\beta_{0}$ & before 2021/09/13 & $2021 / 09 / 13-2021 / 11 / 01$ & after 2021/11/01 \\
$\kappa=2, \beta=1.2 \beta_{0}$ & - & before 2021/09/24 & after 2021/09/24 \\
$\kappa=2, \beta=1.5 \beta_{0}$ & - & - & after 2021/09/01 \\
\hline
\end{tabular}

strong ADE degree, it's hard to have a period during which introducing infected cases is of low risk. However, with strict normalized control interventions (small transmission rate, for example $\beta=0.4 \beta_{0}$ ), even if ADE is slightly feasible ( $\kappa$ varies from 1 to 1.5 ), the emerging outbreak of introducing infected cases would maintain in a low risk till December 31, 2022. From Fig. 10 (c) and (d), we can see that the increasing of the waning rate $\omega$ also leads to the decrease of $T_{1}$ and $T_{2}$. When the transmission rate increases to $0.8 \beta_{0}$, a reduced immunity waning rate $\omega=1 / 180$, can also ensure the emerging outbreak in a low risk by introducing infected cases by the end of the year 2022. However, when the transmission rate is small enough, corresponding to the strict normalized control strategies, the emerging outbreak of introducing infected cases is always maintained in a low risk till December 31,2022, regardless of the waning rate. Both contour plots illustrated that strengthening normalized control interventions is quite efficient in protecting the community from the rapidly outbreak induced by the imported infected cases.

\section{Discussion}

Creating the herd immunity by using COVID-19 vaccines in the population has been taken as the only solution to stop the COVID-19 pandemic for a long time. Especially for the counties or territories experienced multiple epidemic waves even with various non-pharmaceutic interventions (NPIs). We should be sure that vaccines do work to slow down the epidemics. However, immunity waning and ADE may destroy the hope of building the herd immunity to finally eradiate SARS-CoV-2. Therefore, it's urgent and critical to evaluate how long the current vaccination program can protect the country in a low level of risk in the presence of immunity waning, and ADE. This can provide important decision making-basis for the decision makers to determine when the catch-up vaccination program should be launched.

In the current study, we take mainland China as the example to illustrate the possible transmission dynamics of COVID-19 with the current vaccination program. Incorporating the immunity waning dynamics and ADE effect, we developed a new mathematical model. The proposed 
medRxiv preprint doi: https://doi.org/10.1101/2021.08.25.21262601; this version posted August 31, 2021. The copyright holder for this preprint (which was not certified by peer review) is the author/funder, who has granted medRxiv a license to display the preprint in perpetuity.

It is made available under a CC-BY-NC-ND 4.0 International license .
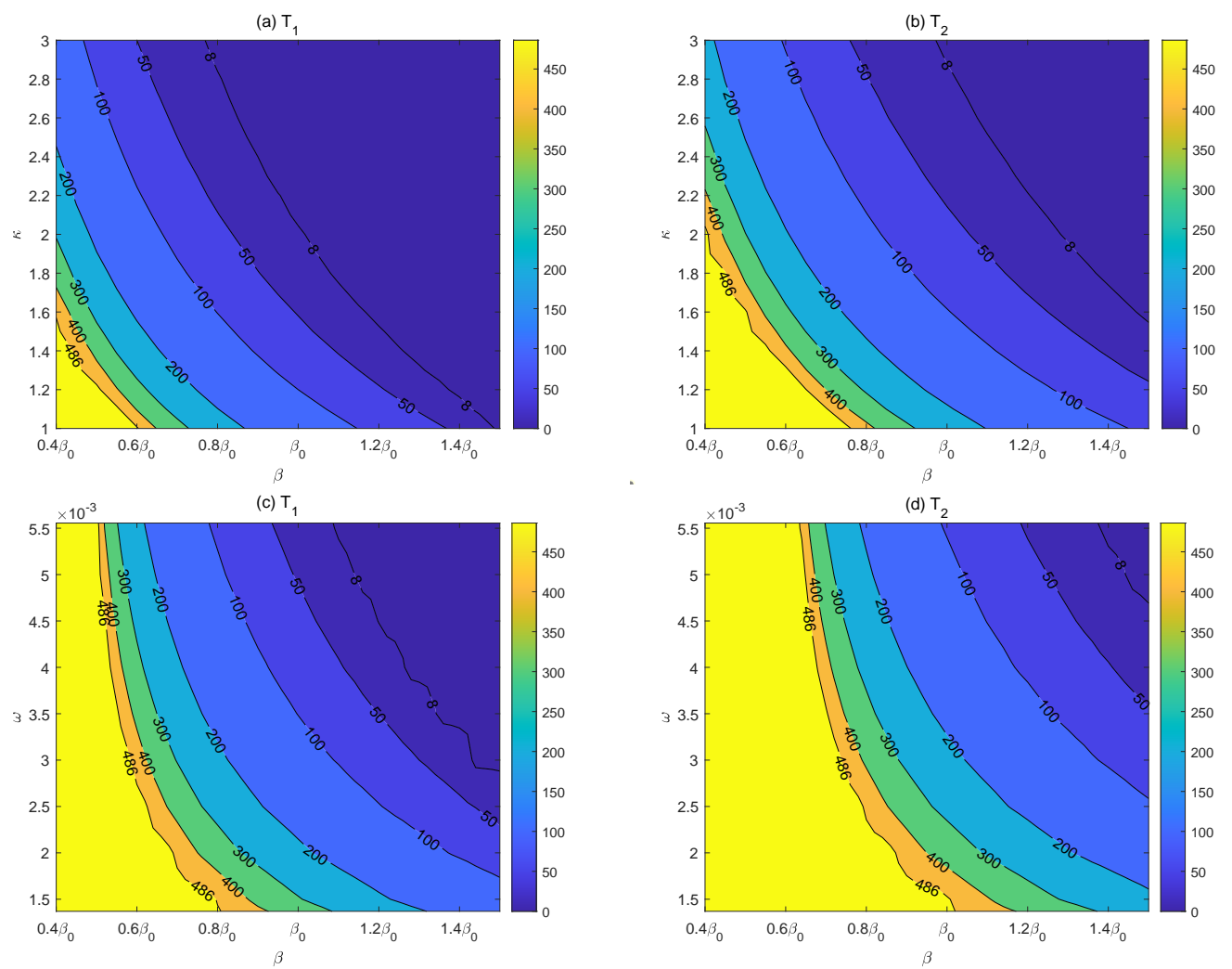

Figure 10: Contour plots of $T_{1}$ and $T_{2}$ with respect to $\beta$ and $\kappa, \beta$ and $\omega$, by taking September 1,2021 as the initial time. 
model was calibrated by using the data of the COVID-19 epidemic in 2020 in mainland China, and the vaccination data from Dec. 15, 2020 to Jun 29, 2021. The estimation showed that the cumulative population with at least one dose reached $56.4 \%$ while the population with $\mathrm{t}$ wo doses reached $32.02 \%$ on June 29, 2021 (the last data point). A prediction indicated that the vaccination coverage with at least one dose would reach $95.87 \%$ and the proportion with two-doses would reach $77.92 \%$ on August 31, 2021. Therefore, the vaccination coverage has already reached a very high level in China, reflecting a spectrum of immunity.

We initially tested if we can return to the pre-COVID-19 pandemic era or not by the mass vaccination program only. That is, we showed if the emerging epidemics, by introducing several new cases into communities, can be under control or not without any other NPIs (i.e. $\beta$ is set to be $\beta_{0}$ ). The answer is definitely no in the presence of immunity waning. We found that the daily reported cases can grow exponentially in a short period since the introducing time, and peak at a huge number. This is directly due to the immunity waning, and a large proportion of vaccinated individuals become susceptible again. We can intuitively see the reason from the newly defined reproduction number $R_{S}$, i.e. the reproduction number at the introducing time. $R_{S}$ increases and exceeds the threshold of unit as the introducing time postpones, attributing to the dynamic of immunity waning in the population. That's also the reason why the introducing time of infected cases has a great influence on the transmission dynamics of COVID-19 epidemics (6). Generally, the later of the introducing time, the shorter period that the daily reported cases take to peak. This indicates that the faster implication of interventions should be considered when the introducing time is long from the stopping time of the vaccination program. We observed an interesting phenomenon that the peak value of the outbreak is non-monotonous with respect to the introducing time.

We also observed from Fig. 5 and 6 that intermittent outbreaks of COVID-19 can occur. Initially, the immunity waning leads to the breakthrough of the herd immunity, consequently, introducing new infected cases can result in a large outbreak. In return, the repeated outbreaks can further boost the herd immunity in the population level and drive the decline of the effective reproduction number, subsequently drive the decline of the epidemics. In conclusion, a loop of immunity waning and immunity boosting in the population induced the intermittent epidemic. Furthermore, it should be mentioned that the amplitudes of the subsequential outbreaks are decreasing over time. This means that a large proportion of the population will be protected sufficiently after several outbreaks. This result implies that boosting the immunity by a booster injection of vaccine in the population may be helpful in mitigating the possible outbreaks. The optimized boosting program should be further studied.

Although the reproduction number can be an important index to represent if the number of newly infected cases is increasing or not, it doesn't mean that the epidemic will be out of control when the effective reproductions number is greater than the unit, as it can be just slightly greater than the unit in a relatively long period. Therefore, from another point of view, we tried to find a new index to represent whether the emerging epidemic is under control. That is, for any given number of cases $\left(I_{0}\right)$ introduced into the community, we say if the newly reported cases is always lower than $I_{0}$ in the next 30 days, it's of a low level of risk in terms 
of the introducing time. With our definition, we found that any emerging epidemic of COVID19 by introducing infected cases before Januray 22, 2022 can be in a low level of risk. As we can see, it's very short for the protective period of the vaccination program. For this reason, we assumed $50 \%$ populations to maintain a normalized control interventions by wearing masks, correspondingly, the transmission rate is set to be $0.6 \beta_{0}$. The results showed that the vaccination program incorporating a normalized interventions can prolong the protective period till January 18, 2023.

$\mathrm{ADE}$ is thought to be a big trouble in the development and use of COVID-19 vaccines. We also quantitatively evaluated the impact of ADE on the transmission dynamic of COVID19 with the implementation of the mass vaccination program. The intuitive results are that ADE can bring forward the peak time of an outbreak and greatly increase the peak value of the daily reported cases. Furthermore, ADE can also increase the frequency of the intermittent outbreaks. In our definition, with a normalized control intervention, ADE can bring forward the critical time of escaping the low risk almost one year (i.e. from January 18, 2023 to January 4, 2022), as listed in Table 2. Considering the higher transmission ability of the SARS-CoV-2 variants, we obtained the similar results as those of ADE. Apparently, ADE and the emerging of new variants have made the control of COVID-19 epidemics even more difficult.

\section{Conclusion}

Utilizing mathematical model, this study focused on investigating the resurgence risk of COVID-19 after the mass vaccination program in China in the presence of immunity waning and ADE. The vaccination coverage is projected to be very high till Aug. 31, 2021, which would almost reach the requested critical level of herd immunity. However, the herd immunity can be easily breakthrough by immunity waning. Therefore, we suggest to maintain a normalized control interventions of wearing masks in the long future even with the mass vaccination program. By defining the risk level of an emerging outbreak, our results showed that the current vaccination program incorporating the normalized interventions can protect China in a low level of resurgence risk till 2023/01/18. However, the emerging evidence of the existence of ADE and the SARS-CoV-2 variants with higher transmission ability have made the situation worse. Therefore, we should get ready for a long struggle with COVID-19, and should not totally rely on COVID-19 vaccines.

It's worth mentioning that boosting the immunity in the population might be able to mitigate the emerging outbreaks. Maintaining the normalized NPIs and booster injection of vaccination periodically could be a good choice in combating with COVID-19 in the long future. How to optimize the periodic vaccination program incorporating the implementation of NPIs is of great significance, which falls within the scope of our further work.

\section{Acknowledgements}

The authors are supported by the National Natural Science Foundation of China (grant numbers: 12031010(ST),11631012(YX),12001349(WZ)), the Postdoctoral Research Foundation of 
China (grant number: 2019M663611 (WZ)), and the Young Talent Support Plan of Xian Jiaotong University (BT).

\section{References}

\section{References}

[1] World Health Organization, Coronavirus disease (covid-19): Vaccines, https://www . who.int/news-room/q-a-detail/coronavirus-disease-(covid-19)-vaccines (2021).

[2] National Health Commission of the People's Republic of China, Over 1.82 bln doses of covid-19 vaccines administered in china, http://en.nhc.gov.cn/2021-08/13/c_ 84379.htm (2021).

[3] World Health Organization, Who coronavirus (covid-19) dashboard, https : //covid19. who . int/table (2021).

[4] V. Shinde, S. Bhikha, Z. Hoosain, M. Archary, Q. Bhorat, L. Fairlie, U. Lalloo, M. S. Masilela, D. Moodley, S. Hanley, et al., Efficacy of nvx-cov2373 covid-19 vaccine against the b. 1.351 variant, New England Journal of Medicine 384 (20) (2021) 1899-1909.

[5] P. G. Choe, C. K. Kang, H. J. Suh, J. Jung, K.-H. Song, J. H. Bang, E. S. Kim, H. B. Kim, S. W. Park, N. J. Kim, et al., Waning antibody responses in asymptomatic and symptomatic sars-cov-2 infection, Emerging infectious diseases 27 (1) (2021) 327.

[6] W. N. Chia, F. Zhu, S. W. X. Ong, B. E. Young, S.-W. Fong, N. Le Bert, C. W. Tan, C. Tiu, J. Zhang, S. Y. Tan, et al., Dynamics of sars-cov-2 neutralising antibody responses and duration of immunity: a longitudinal study, The Lancet Microbe 2 (6) (2021) e240-e249.

[7] Y. Liu, W. T. Soh, J.-i. Kishikawa, M. Hirose, E. E. Nakayama, S. Li, M. Sasai, T. Suzuki, A. Tada, A. Arakawa, et al., An infectivity-enhancing site on the sars-cov-2 spike protein targeted by antibodies, Cell 184 (2021) 1-15.

[8] S. B. Halstead, Pathogenesis of dengue: challenges to molecular biology, Science 239 (4839) (1988) 476-481.

[9] W. Dejnirattisai, A. Jumnainsong, N. Onsirisakul, P. Fitton, S. Vasanawathana, W. Limpitikul, C. Puttikhunt, C. Edwards, T. Duangchinda, S. Supasa, et al., Cross-reacting antibodies enhance dengue virus infection in humans, Science 328 (5979) (2010) 745-748.

[10] W. Ndifon, N. S. Wingreen, S. A. Levin, Differential neutralization efficiency of hemagglutinin epitopes, antibody interference, and the design of influenza vaccines, Proceedings of the National Academy of Sciences 106 (21) (2009) 8701-8706. 
[11] W. Dejnirattisai, P. Supasa, W. Wongwiwat, A. Rouvinski, G. Barba-Spaeth, T. Duangchinda, A. Sakuntabhai, V.-M. Cao-Lormeau, P. Malasit, F. A. Rey, et al., Dengue virus sero-cross-reactivity drives antibody-dependent enhancement of infection with zika virus, Nature immunology 17 (9) (2016) 1102-1108.

[12] L. Priyamvada, K. M. Quicke, W. H. Hudson, N. Onlamoon, J. Sewatanon, S. Edupuganti, K. Pattanapanyasat, K. Chokephaibulkit, M. J. Mulligan, P. C. Wilson, et al., Human antibody responses after dengue virus infection are highly cross-reactive to zika virus, Proceedings of the National Academy of Sciences 113 (28) (2016) 7852-7857.

[13] S. V. Bardina, P. Bunduc, S. Tripathi, J. Duehr, J. J. Frere, J. A. Brown, R. Nachbagauer, G. A. Foster, D. Krysztof, D. Tortorella, et al., Enhancement of zika virus pathogenesis by preexisting antiflavivirus immunity, Science 356 (6334) (2017) 175-180.

[14] A. S. Agrawal, X. Tao, A. Algaissi, T. Garron, K. Narayanan, B.-H. Peng, R. B. Couch, C.T. K. Tseng, Immunization with inactivated middle east respiratory syndrome coronavirus vaccine leads to lung immunopathology on challenge with live virus, Human vaccines \& immunotherapeutics 12 (9) (2016) 2351-2356.

[15] C. Y. Cheung, L. L. Poon, I. H. Ng, W. Luk, S.-F. Sia, M. H. Wu, K.-H. Chan, K.-Y. Yuen, S. Gordon, Y. Guan, et al., Cytokine responses in severe acute respiratory syndrome coronavirus-infected macrophages in vitro: possible relevance to pathogenesis, Journal of virology 79 (12) (2005) 7819-7826.

[16] D. O. Ricke, Two different antibody-dependent enhancement (ade) risks for sars-cov-2 antibodies, Frontiers in immunology 12 (2021) 443.

[17] N. Ferguson, R. Anderson, S. Gupta, The effect of antibody-dependent enhancement on the transmission dynamics and persistence of multiple-strain pathogens, Proceedings of the National Academy of Sciences 96 (2) (1999) 790-794.

[18] D. A. Cummings, I. B. Schwartz, L. Billings, L. B. Shaw, D. S. Burke, Dynamic effects of antibody-dependent enhancement on the fitness of viruses, Proceedings of the National Academy of Sciences 102 (42) (2005) 15259-15264.

[19] B. Adams, E. Holmes, C. Zhang, M. Mammen, S. Nimmannitya, S. Kalayanarooj, M. Boots, Cross-protective immunity can account for the alternating epidemic pattern of dengue virus serotypes circulating in bangkok, Proceedings of the National Academy of Sciences 103 (38) (2006) 14234-14239.

[20] B. Tang, Y. Xiao, J. Wu, Implication of vaccination against dengue for zika outbreak, Scientific reports 6 (1) (2016) 1-14.

[21] B. Tang, W. Zhou, Y. Xiao, J. Wu, Implication of sexual transmission of zika on dengue and zika outbreaks, Math. Biosci. Eng 16 (2019) 5092-5113. 
[22] B. Tang, Y. Xiao, B. Sander, M. A. Kulkarni, R.-L. R. Team, J. Wu, et al., Modelling the impact of antibody-dependent enhancement on disease severity of zika virus and dengue virus sequential and co-infection, Royal Society open science 7 (4) (2020) 191749.

[23] P. J. Hotez, D. B. Corry, M. E. Bottazzi, Covid-19 vaccine design: the janus face of immune enhancement, Nature Reviews Immunology 20 (6) (2020) 347-348.

[24] J. A. Tetro, Is covid-19 receiving ade from other coronaviruses?, Microbes and infection 22 (2) (2020) 72-73.

[25] L. Atlani-Duault, B. Lina, F. Chauvin, J.-F. Delfraissy, D. Malvy, Immune evasion means we need a new covid-19 social contract, The Lancet Public Health 6 (4) (2021) e199-e200.

[26] Our World in Data, Coronavirus (covid-19) vaccinations, https://ourworldindata.org/covid-vaccinations\# what-share-of-the-population-has-been-fully-vaccinated-against-covid-19 (2021).

[27] National Health Commission of the People's Republic of China, Infographic: All you need to know about taking covid-19 vaccines, http://en.nhc.gov.cn/2021-04/12/ c_83463.htm (2021).

[28] National Bureau of Statistics of China, Main data of the seventh national population census, http://www.stats.gov.cn/english/PressRelease/202105/t20210510_ 1817185.html (2021).

[29] X. Hao, S. Cheng, D. Wu, T. Wu, X. Lin, C. Wang, Reconstruction of the full transmission dynamics of covid-19 in wuhan, Nature 584 (7821) (2020) 420-424.

[30] J. A. Backer, D. Klinkenberg, J. Wallinga, Incubation period of 2019 novel coronavirus (2019-ncov) infections among travellers from wuhan, china, 20-28 january 2020, Eurosurveillance 25 (5) (2020) 2000062.

[31] Q. Li, X. Guan, P. Wu, Early transmission dynamics in wuhan, china, of novel coronaviruscinfected pneumonia, The New England Journal of Medicine 382 (13) (2020) 11991207.

[32] Sixth Tone, Chinese-developed covid-19 vaccine over $91 \%$ effective, turkey says, https://www.sixthtone.com/news/1006620/ chinese-developed-covid-19-vaccine-over-91\%25-effective\% 2C-turkey-says (2021).

[33] The University of MelBourne, What are the sinopharm and sinovac vaccines? and how effective are they? two experts explain, https://findanexpert.unimelb. edu.au/news/15054-what-are-the-sinopharm-and-sinovac-vaccines\% 3F-and-how-effective-are-they\%3F-two-experts-explain (2021). 
[34] M. D. Tanriover, H. L. Doanay, M. Akova, H. R. Gner, K. Aksu, Efficacy and safety of an inactivated whole-virion sars-cov-2 vaccine (coronavac): interim results of a double-blind, randomised, placebo-controlled, phase 3 trial in turkey, The Lancet 398 (10296) 213-222.

[35] H. J. Wearing, P. Rohani, Ecological and immunological determinants of dengue epidemics, Proceedings of the National Academy of Sciences 103 (31) (2006) 11802-11807.

[36] M. Recker, K. B. Blyuss, C. P. Simmons, T. T. Hien, B. Wills, J. Farrar, S. Gupta, Immunological serotype interactions and their effect on the epidemiological pattern of dengue, Proceedings of the Royal Society B: Biological Sciences 276 (1667) (2009) 2541-2548.

[37] D. K. Chu, E. A. Akl, S. Duda, K. Solo, S. Yaacoub, H. J. Schünemann, A. El-harakeh, A. Bognanni, T. Lotfi, M. Loeb, et al., Physical distancing, face masks, and eye protection to prevent person-to-person transmission of sars-cov-2 and covid-19: a systematic review and meta-analysis, The lancet 395 (10242) (2020) 1973-1987.

[38] S. Marino, I. B. Hogue, C. J. Ray, D. E. Kirschner, A methodology for performing global uncertainty and sensitivity analysis in systems biology, Journal of theoretical biology 254 (1) (2008) 178-196.

[39] J. Wu, B. Tang, Y. Xiao, S. Tang, A. Ahmad, J. Orbinski, Swift mitigations and tipping point cascade effect: Rethinking covid-19 control and prevention measures to prevent and limit future outbreaks, Health Management, Policy and Innovation 5 (1) (2020) special issue on COVID-19. 


\section{Supplementary Information: The resurgence risk of COVID-19 in the presence of immunity waning and ADE effect: a mathematical modelling study}

August 25, 2021

\section{The model}

\section{Full structure model}

The full modelling framework includes the infection and transmission dynamic of COVID-19 and the two-doses vaccination program and immunity waning dynamic.

Transmission progression: We used an SEIARS model structure to describe the transmission dynamics of COVID-19. That is, according to the epidemic status of COVID-19 infections, the total population is divided into susceptible $(S)$, exposed $(E)$, symptomatic infected $(I)$, asymptomatic infected $(A)$, recovered $(R)$ classes $[2,3,4]$. The susceptible population will enter into the exposed class $(E)$ once they are infected by symptomatic or asymptomatic infected population. The exposed individuals move to $I$ or $A$ at a rate of $\sigma$. And we assume that the probability of showing symptoms is $\rho$. The recovery rates of $I$ and $A$ are set to be $\gamma_{I}$ and $\gamma_{A}$, respectively. Given the immunity waning, the recovered population can become susceptible again. The transmission diagram with the mass vaccination program is shown in Fig. 1.

Vaccination program for susceptible population: Susceptible population $(S)$, will be firstly vaccinated by onedose at a rate $v_{1}$. The effective protection rate by one-dose is $p_{1}$, hence, the part $p_{1} v_{1}$ can be effectively protected and temporarily immune to COVID-19, and the class is denoted by $V_{1}$. The rest part $\left(\left(1-p_{1}\right) v_{1}\right)$ remains in the susceptible class, which is denoted by $S_{V_{1}}$. The population vaccinated by one-dose will be further vaccinated by a second dose after a pre-set period since the first dose. Similarly, we set a protection rate of the second dose as $p_{2}$. Once vaccinated by the second dose, individuals will either move to the class effectively protected (denoted by $V_{2}$ ), or to the class susceptible to COVID-19 (denoted by $S_{V_{2}}$ ). We assume that the population effectively protected by 
one-dose (the persons in $V_{1}$ ), will all move to $V_{2}$ when they receive the second dose. It should be mentioned that the population vaccinated but not immune to COVID-19 $\left(S_{V_{1}}, S_{V_{2}}\right)$ can be infected. Then according to the epidemic status, we further have the classes of $E_{j}, I_{j}, A_{j}, R_{j}, j \in\left\{V_{1}, V_{2}\right\}$ as shown in Fig. 1.

Immunity waning and ADE effects: As we mentioned in the introduction, lots of evidence suggest that the neutralizing antibodies decay significantly since the onset of symptoms of COVID-19 patients, indicating the existence of immunity waning. Given the immunity waning, we assume that both the recovered and effectively vaccinated population can temporarily immune to COVID-19, and will return back to susceptible classes. In particularly, we assume that the individuals in $V_{1}$ will move to $S_{V_{1 \omega}}$ due to the waning of immunity, and the population in $R, R_{V_{1}}, R_{V_{2}}, V_{2}$ will move to $S_{V_{2 \omega}}$. Correspondingly, the rate of immunity waning are denoted by $\omega_{i}, i \in\left\{R, R_{V_{1}}, R_{V_{2}}, V_{1}, V_{2}\right\}$. On the other hand, as the decay of the neutralizing antibodies, the binding antibodies can dominant the immune response, which will enhance the infectivity of the virus (i.e. ADE effects). That is, compared with other susceptible population, the susceptibility of $S_{V_{1 \omega}}$ and $S_{V_{2 \omega}}$ is much higher as the pre-existing of immunity. Here, we denote $\kappa$ as the modification factor of the susceptibility of the individuals who have lost their immunity.

Based on the above assumptions, the model equations can be written as: 


$$
\begin{aligned}
& \frac{d S}{d t} \quad=-\beta S \frac{I+I_{V_{1}}+I_{V_{2}}+\theta\left(A+A_{V_{1}}+A_{V_{2}}\right)}{N}-v_{1} S, \\
& \frac{d E}{d t}=\beta S \frac{I+I_{V_{1}}+I_{V_{2}}+\theta\left(A+A_{V_{1}}+A_{V_{2}}\right)}{N}-\sigma E, \\
& \frac{d I}{d t}=\rho \sigma E-\gamma_{I} I-\delta_{I} I, \\
& \frac{d A}{d t} \quad=(1-\rho) \sigma E-\gamma_{A} A, \\
& \frac{d H}{d t}=\delta_{I} I-\gamma_{H} H-\alpha H, \\
& \frac{d R}{d t}=\gamma_{A} A+\gamma_{I} I+\gamma_{H} H-\omega_{R} R, \\
& \frac{d V_{1}}{d t}=p_{1} v_{1} S-v_{2} V_{1}-\omega_{V_{1}} V_{1} \\
& \frac{d V_{2}}{d t}=v_{2} V_{1}+p_{2} v_{2} S_{V_{1}}+p_{3} v_{2} S_{V_{1 \omega}}-\omega_{V_{2}} V_{2}, \\
& \frac{d S_{V_{1 \omega}}}{d t}=\omega_{V_{1}} V_{1}-\kappa \beta S_{V_{1 \omega}} \frac{I+I_{V_{1}}+I_{V_{2}}+\theta\left(A+A_{V_{1}}+A_{V_{2}}\right)}{N}-v_{2} S_{V_{1 \omega}} \text {, } \\
& \frac{d S_{V_{2 \omega}}}{d t}=-\kappa \beta S_{V_{2 \omega}} \frac{I+I_{V_{1}}+I_{V_{2}}+\theta\left(A+A_{V_{1}}+A_{V_{2}}\right)}{N}+\omega_{R_{V_{1}}} R_{V_{1}}+\omega_{R_{V_{2}}} R_{V_{2}}+\omega_{R} R+\omega_{V_{2}} V_{2} \text {, } \\
& \frac{d S_{V_{1}}}{d t}=\left(1-p_{1}\right) v_{1} S-\beta S_{V_{1}} \frac{I+I_{V_{1}}+I_{V_{2}}+\theta\left(A+A_{V_{1}}+A_{V_{2}}\right)}{N}-v_{2} S_{V_{1}} \text {, } \\
& \frac{d E_{V_{1}}}{d t}=\left(\kappa \beta S_{V_{1 \omega}}+\beta S_{V_{1}}\right) \frac{I+I_{V_{1}}+I_{V_{2}}+\theta\left(A+A_{V_{1}}+A_{V_{2}}\right)}{N}-\sigma E_{V_{1}}, \\
& \frac{d I_{V_{1}}}{d t}=\rho \sigma E_{V_{1}}-\gamma_{I} I_{V_{1}}-\delta_{I} I_{V_{1}} \\
& \frac{d A_{V_{1}}}{d t}=(1-\rho) \sigma E_{V_{1}}-\gamma_{A} A_{V_{1}} \text {, } \\
& \frac{d H_{V_{1}}}{d t}=\delta_{I} I_{V_{1}}-\gamma_{H} H_{V_{1}}-\alpha H_{V_{1}} \text {, } \\
& \frac{d R_{V_{1}}}{d t}=\gamma_{A} A_{V_{1}}+\gamma_{I} I_{V_{1}}+\gamma_{H} H_{V_{1}}-\omega_{R_{V_{1}}} R_{V_{1}} \text {, } \\
& \frac{d S_{V_{2}}}{d t}=\left(1-p_{2}\right) v_{2} S_{V_{1}}+\left(1-p_{3}\right) v_{2} S_{V_{1 \omega}}-\beta S_{V_{2}} \frac{I+I_{V_{1}}+I_{V_{2}}+\theta\left(A+A_{V_{1}}+A_{V_{2}}\right)}{N} \\
& \frac{d E_{V_{2}}}{d t}=\left(\kappa \beta S_{V_{2 \omega}}+\beta S_{V_{2}}\right) \frac{\left(I+I_{V_{1}}+I_{V_{2}}+\theta\left(A+A_{V_{1}}+A_{V_{2}}\right)\right)}{N}-\sigma E_{V_{2}}, \\
& \frac{d I_{V_{2}}}{d t}=\rho \sigma E_{V_{2}}-\gamma_{I} I_{V_{2}}-\delta_{I} I_{V_{2}} \\
& \frac{d A_{V_{2}}}{d t}=(1-\rho) \sigma E_{V_{2}}-\gamma_{A} A_{V_{2}}, \\
& \frac{d H_{V_{2}}}{d t}=\delta_{I} I_{V_{2}}-\gamma_{H} H_{V_{2}}-\alpha H_{V_{2}}, \\
& \frac{d R_{V_{2}}}{d t}=\gamma_{A} A_{V_{2}}+\gamma_{I} I_{V_{2}}+\gamma_{H} H_{V_{2}}-\omega_{R_{V_{2}}} R_{V_{2}} .
\end{aligned}
$$

Here, $N$ is the whole population in the considered region. The equations for $S, E, I, A, H, R$ represent the infection and transmission dynamic in the non-vaccinated population, the equations for $S_{V_{1}}, E_{V_{1}}, I_{V_{1}}, A_{V_{1}}, H_{V_{1}}, R_{V_{1}}$ represent the infection and transmission dynamic in the population vaccinated with the first dose vaccine, the equations for $S_{V_{2}}, E_{V_{2}}, I_{V_{2}}, A_{V_{2}}, H_{V_{2}}, R_{V_{2}}$ represent the infection and transmission dynamic in the population vaccinated with two doses vaccine. $V_{1}$ and $V_{2}$ are the population effectively protected by the first dose and two doses vaccine, respectively, who would not evolve in the transmission dynamic but will lose the immunity with rate $\omega_{V_{1}}$ and $\omega_{V_{2}}$, respectively. $S_{V_{1 \omega}}, S_{V_{2 \omega}}$ are the population lost the immunity and are more susceptible to the infection due to ADE effect. The detailed definitions of all the parameters are listed in Table 1.

Using the next generation matrix method, we can firstly calculate the basic reproduction number [1]. Then subsisting the time-varying parameters and variables into the basic reproduction number, we can obtain the effective 
reproduction number of model (1), which is given by

$$
R_{t}=R(t)=\beta\left(\frac{\rho}{\delta_{I}+\gamma_{I}}+\frac{\theta(1-\rho)}{\gamma_{A}}\right) \frac{S(t)+S_{v_{1}}(t)+S_{V_{2}}(t)+\kappa S_{V_{1 \omega}}(t)+\kappa S_{V_{2 \omega}}(t)}{N(t)} .
$$

\section{Transmission dynamic model without vaccination dynamic}

Note that, during the outbreak of COVID-19 in mainland China in 2020, there is no vaccination, consequently, model (1) can be reduced to

$$
\begin{aligned}
\frac{d S}{d t} & =-\beta(t) S \frac{I+\theta A}{N} \\
\frac{d E}{d t} & =\beta(t) S \frac{I+\theta A}{N}-\sigma E \\
\frac{d I}{d t} & =\rho \sigma E-\delta_{I}(t) I-\gamma_{I} I \\
\frac{d A}{d t} & =(1-\rho) \sigma E-\gamma_{A} A \\
\frac{d H}{d t} & =\delta_{I}(t) I-\gamma_{H} H-\alpha H \\
\frac{d R}{d t} & =\gamma_{I} I+\gamma_{A} A+\gamma_{H} H .
\end{aligned}
$$

Here, considering the continuously enhanced control interventions by the government, we introduced two timedependent parameters into the system. In detail, the transmission rate $\beta$ is set to be a decreasing function of time $t$ with the following form

$$
\beta(t)=\left(\beta_{0}-\beta_{1}\right) e^{-r_{b} t}+\beta_{1}
$$

where $\beta_{0}$ is the initial transmission rate, $\beta_{1}$ is the minimum transmission rate, and $r_{b}$ is the exponential decreasing rate of the transmission rate. Similarly, we set the diagnose rate is a increasing function of time $t$ with the following form

$$
\delta_{I}(t)=\left(\delta_{I_{0}}-\delta_{I_{1}}\right) e^{-r_{d} t}+\delta_{I_{1}}
$$

where $\delta_{I_{0}}$ is the initial diagnose rate, $\delta_{I_{1}}$ is the maximum diagnose rate, and $r_{d}$ is the exponential increasing rate of the diagnose rate. $t_{0}$ corresponds to January 23, 2020.

Model (3) with (4) and (5) can be used to describe the transmission dynamic of the COVID-19 epidemics in mainland China in 2020, with the gradually improved and enhanced non-pharmaceutic interventions (NPIs), and without vaccination. Therefore, model (3) can be used to fit the epidemic data of COVID-19 epidemic in mainland China in 2020, to obtain the values of parameters related to the disease transmission.

\section{Vaccination dynamic model without transmission dynamic}

Since the initiation of the vaccination program, it has already taken more than 9 months to vaccinate against COVID-19 in mainland China. Therefore, the vaccination program is also a dynamic process. To model the vaccination dynamic without the transmission dynamic, a three-compartment model reduced from model (1) was 
derived. The three compartments are the population without vaccination $(S)$, the population got the first vaccine dose $\left(S_{1}\right)$ and the population who have received the second vaccine dose $\left(S_{2}\right)$, respectively. Then the model is given by:

$$
\begin{aligned}
\frac{d S}{d t} & =-v_{1}(t) S, \\
\frac{d S_{1}}{d t} & =v_{1}(t) S-v_{2} S_{1}, \\
\frac{d S_{2}}{d t} & =v_{2} S_{1} .
\end{aligned}
$$

where $v_{1}(t)$ is the time-dependent vaccination rate, as it should be small initially since the availability of the number of vaccine doses was limited at the beginning, and then exponentially increased as the production of COVID19 vaccines was accelerated, and finally it could plateau to a constant level depending on the daily vaccination capacity. Thus, we assume $v_{1}(t)$ as a logistic increasing function of time $t$ with the following form:

$$
v_{1}(t)=\frac{v_{0} v_{b}}{v_{0}+\left(v_{b}-v_{0}\right) e^{-r_{v} t}}
$$

with $t$ corresponds to the time when the initial time is assumed to be December 15, 2020. As a result, model (6) can be used to fit the vaccination data in mainland China, to obtain the values of parameters related to the vaccination program.

\section{Immunity waning dynamic model without transmission dynamic}

Note that immunity waning makes the people who have gained the immunity after vaccination become susceptible again, thus based on model (1) and incorporating the natural immunity waning into model (6), we can obtain the following system:

$$
\begin{aligned}
\frac{d S}{d t} & =-v_{1} S, \\
\frac{d V_{1}}{d t} & =p_{1} v_{1} S-v_{2} V_{1}-\omega_{V_{1}} V_{1}, \\
\frac{d V_{2}}{d t} & =v_{2} V_{1}+p_{2} v_{2} S_{V_{1}}+p_{3} v_{2} S_{V_{1 \omega}}-\omega_{V_{2}} V_{2}, \\
\frac{d S_{V_{1}}}{d t} & =\omega_{V_{1}} V_{1}-v_{2} S_{V_{1} \omega}, \\
\frac{d S_{V_{2 \omega}}}{d t} & =\omega_{V_{2}} V_{2}, \\
\frac{d S_{V_{1}}}{d t} & =\left(1-p_{1}\right) v_{1} S-v_{2} S_{V_{1}}, \\
\frac{d S_{V_{2}}}{d t} & =\left(1-p_{2}\right) v_{2} S_{V_{1}}+\left(1-p_{3}\right) v_{2} S_{V_{1 \omega}} .
\end{aligned}
$$

Then, model (8) combined the vaccination dynamic and the nature immunity wanning dynamic, but without the transmission dynamic of COVID-19. That is, model (8) can be used to simulate the vaccination dynamics and the immunity waning dynamic during the pre-epidemic period. 


\section{Theoretical illustration of $T_{1}$ and $T_{2}$ remain constant with different $I_{0}$}

We will briefly illustrate that the time length it takes for the number of newly confirmed cases reaching the level of initial introducing infected cases is independent of the initial value of the introducing infected cases, by using a simple SEIAHR model in the following.

Assume that several infected cases $\left(I_{0}\right)$ are introduced at time $t_{0}$ in a fully susceptible population, then the system

$$
\begin{aligned}
\frac{d S}{d t} & =-\frac{\beta S(I+\theta A)}{N}, \\
\frac{d E}{d t} & =\frac{\beta S(I+\theta A)}{N}-\sigma E, \\
\frac{d I}{d t} & =\rho \sigma E-\delta_{I} I-\gamma_{I} I, \\
\frac{d A}{d t} & =(1-\rho) \sigma E-\gamma_{A} A, \\
\frac{d H}{d t} & =\delta_{I} I-\gamma_{H} H-\alpha H, \\
\frac{d R}{d t} & =\gamma_{I} I+\gamma_{A} A+\gamma_{H} H .
\end{aligned}
$$

has the initial condition $\left(S\left(t_{0}\right), E\left(t_{0}\right), I\left(t_{0}\right), A\left(t_{0}\right), H\left(t_{0}\right), R\left(t_{0}\right)\right)=\left(S_{0}, 0, I_{0}, 0,0,0\right)$ at the initial time $t_{0}$, and $I_{0} \ll N_{0}$. Thus it is reasonable to assume $\frac{S}{N} \approx 1$ in the initial stage of the disease transmission, then by omitting the equation of $S$ and $R$, the model can be reduced to

$$
\begin{aligned}
& \frac{d E}{d t}=\beta(I+\theta A)-\sigma E, \\
& \frac{d I}{d t}=\rho \sigma E-\delta_{I} I-\gamma_{I} I, \\
& \frac{d A}{d t}=(1-\rho) \sigma E-\gamma_{A} A, \\
& \frac{d H}{d t}=\delta_{I} I-\gamma_{H} H-\alpha H,
\end{aligned}
$$

with initial condition $\left(E\left(t_{0}\right), I\left(t_{0}\right), A\left(t_{0}\right), H\left(t_{0}\right)\right)=\left(0, I_{0}, 0,0\right)$. Denote $e=\frac{E}{I_{0}}, i=\frac{I}{I_{0}}, a=\frac{A}{I_{0}}, h=\frac{H}{I_{0}}$, system 10 , is equivalent to the following system

$$
\begin{aligned}
\frac{d e}{d t} & =\beta(i+\theta a)-\sigma e, \\
\frac{d i}{d t} & =\rho \sigma e-\delta_{I} i-\gamma_{I} i \\
\frac{d a}{d t} & =(1-\rho) \sigma e-\gamma_{A} a, \\
\frac{d h}{d t} & =\delta_{I} i-\gamma_{H} h-\alpha h,
\end{aligned}
$$

with initial value $(0,1,0,0)$. Thus the solution of system 11 is independent of the value of $I_{0}$ and is unique. Thus $t_{1}$ is constant regardless the value of $I_{0}$, where $i\left(t_{1}\right)=1 / \delta$, namely $\delta I\left(t_{1}\right)=I_{0}$. Thus illustrating that the time it takes for the newly confirmed cases $\delta I$ increases to the initial value $I_{0}$ is independent of $I_{0}$. 


\section{References}

[1] Driessche P, Watmough J. Reproduction numbers and sub-threshold endemic equilibria for compartmental models of disease transmission. Math Biosci, 2002, 180, 29-48.

[2] Tang B, Wang X, Li Q, et al. Estimation of the transmission risk of the 2019-nCoV and its implication for public health interventions. J Clin Med, 2020, 9: 462.

[3] Tang B, Bragazzi N, Li Q, et al. An updated estimation of the risk of transmission of the novel coronavirus (2019-nCov). Infect Dis Model, 2020, 5:248-255.

[4] Tang B, Xia F, Tang S Y, et al. The effectiveness of quarantine and isolation determine the trend of the COVID-19 epidemics in the final phase of the current outbreak in China. Int J Infect Dis, 2020, 95: 288-293. 